\title{
THE EFFECT OF DENERVATION ON NAIL GROWTH*
}

\author{
BY
}

\section{S. SUNDERLAND and L. J. RAY}

\section{From the Department of Anatomy and Histology, University of Melbourne, Australia}

Weir Mitchell (1872) and Sharpey-Schafer (1931) reported that section of a nerve supplying cutaneous fibres to the fingers results in a retardation of nail growth. Head and Sherren (1905), however, believed that the slowing of growth observed in these cases was due entirely to the immobilization of the limb either as a result of splinting or paralysis and that division of sensory fibres without limitation of digital movements is not associated with any deficiency in nail growth. According to Bernhardt (1881) " nail growth is relatively independent of the influence of the nerve fibres innervating the fingers and tissues of the nail bed ", though his method of measuring growth from "the upper margin of the epidermis covering the nail root" was open to error. In view of these few and contradictory observations it was decided to investigate nail growth in a series of patients with peripheral nerve injuries.

\section{Material and Methods}

Observations on the effects of denervation on nail growth were made on 10 patients with peripheral nerve injuries (Table I). In three of these the ulnar nerve and in six the median nerve had been severed and repaired. One patient sustained a crush injury of the right median nerve when it was caught between the jaws of a Spencer Wells forceps applied to a lacerated brachial artery in a wound in the upper arm. This resulted in completely interrupting conduction in the fibres innervating the digits for more than six months. In five of the median nerve injuries there was a concomitant injury of the brachial artery which necessitated ligation of that vessel. This introduced a complicating vascular factor at the periphery of the limb which, despite the establishment of an adequate collateral circulation, may have contributed to deviations from the normal rate of growth which were observed in these cases.

For data relating to the rate of nail growth in normal digits under a variety of conditions and to the range of normal variation in that growth we

* This work was assisted by a grant from the National Health and Medical Research Council of Australia. have relied on the valuable paper by Le Gros Clark and Buxton (1938). Our technique for measuring nail growth was substantially the same as that advocated and used by them except that we did not attempt a degree of accuracy in measurement greater than 0.25 of a millimetre which we found suitable for the purposes of our enquiry. Readings in most cases were made at weekly intervals. As one mark on the nail advanced distally, another was made at a specified distance from the margin of the lunula. Thus the number of recordings registered in column 4 of Table I refers to the number of marks which were made and traced distally.

The difficulty of obtaining suitable clinical material for prolonged and unbroken observations explains the small numbers of cases available for the enquiry. However, these have been investigated in some detail and cover all seasons of the year.

Observations were made on the nails of the thumb, index, and little fingers. The index and little fingers provide the most dependable readings because the nail beds of these digits are innervated solely by the median (index) and ulnar (little) nerves. The ulnar nerve distribution in the hand commonly includes part or all of the ring finger and may extend to involve the middle finger while the nail bed of the thumb is innervated by the superficial radial nerve as well as by the median. For these reasons we attach importance only to the data provided by measurements of nail growth of the index and little fingers following lesions of the median and ulnar nerves respectively.

\section{Results}

Relevant data relating to the nerve injury sustained by the patients investigated are given in Table $I$. Details relating to the growth of the nails in the denervated and non-denervated digits are given in Tables I and II.

\section{Discussion}

An analysis of the data recorded justifies the following statements. 
TABLE I

RATE OF GROWTH IN DENERVATED AND NON-DENERVATED DIGITS

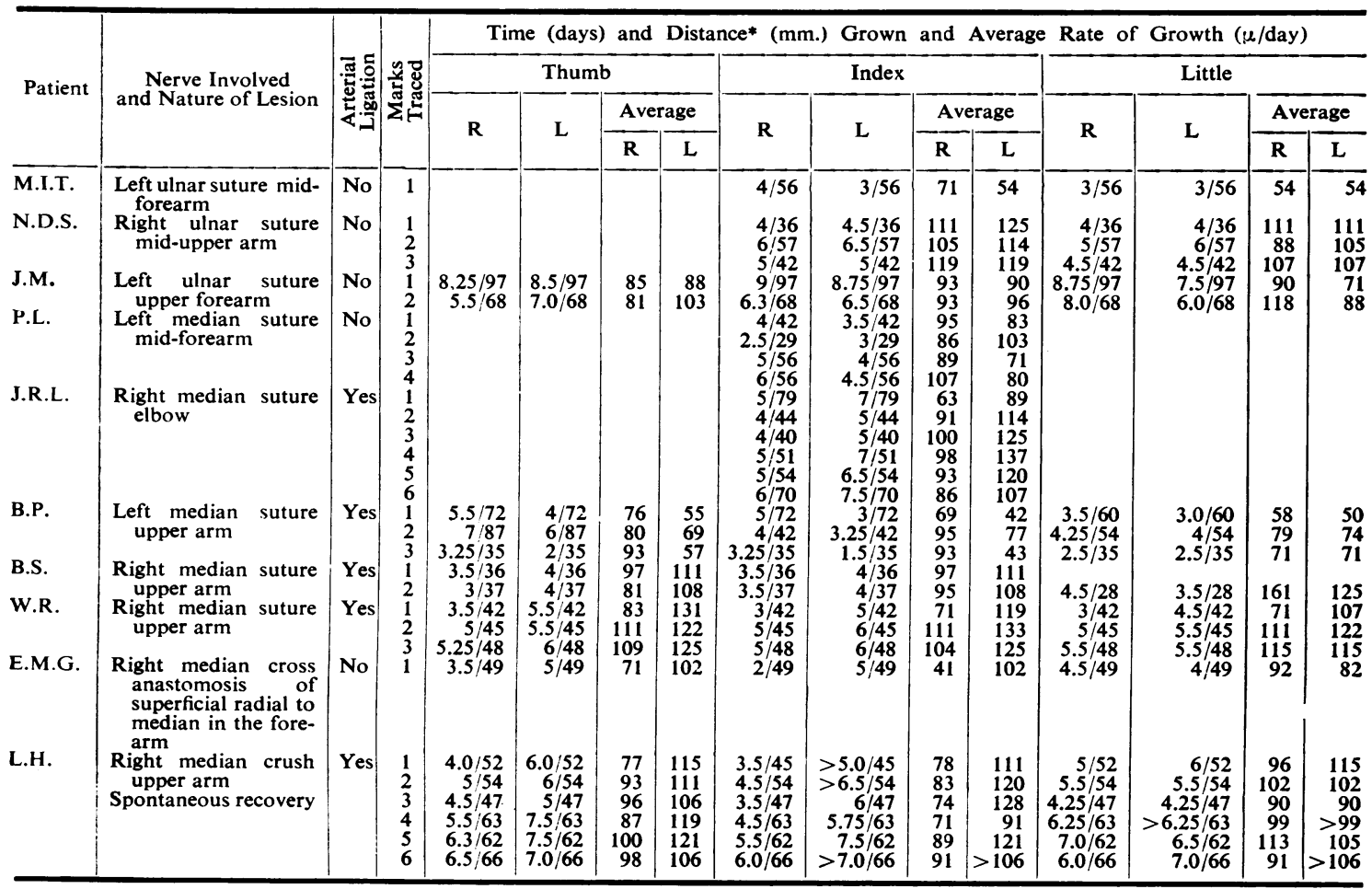

*The value for distance precedes that for time.

TABLE II

AVERAGE RATE OF GROWTH FOR FULL PERIOD OF EACH INVESTIGATION

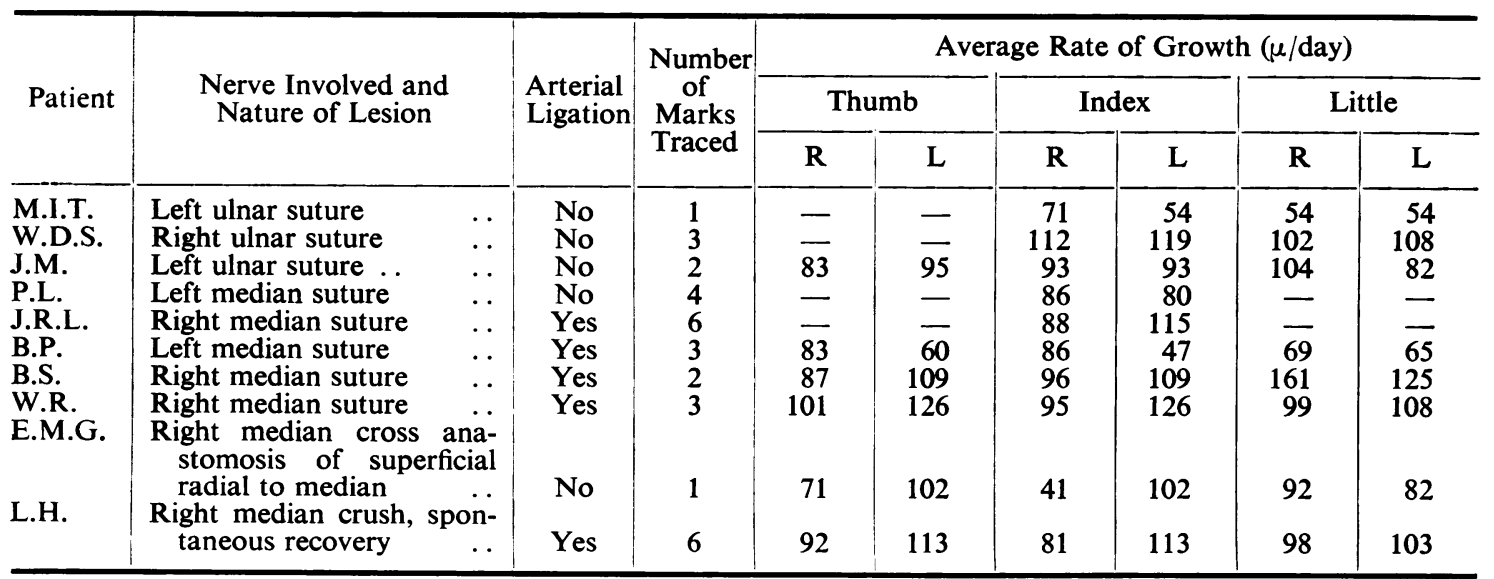

Rate of Growth in Nerve Lesions Unassociated with Ligation of the Main Artery to the Limb.Uncomplicated denervation of the digits had a variable effect on nail growth. The denervation was without effect in one patient, a questionable slowing was recorded in two, and in a fourth growth was slightly but definitely retarded. In another patient, who had sustained an irreparable injury 
of the median nerve, the affected hand was the seat of articular and periarticular changes and trophic disturbances. In this case there was a most pronounced slowing of nail growth in the thumb and index finger while growth in the little finger proceeded normally.

It is unlikely that the retardation recorded could be attributed to immobilization. The affected parts were not splinted and were subjected to frequent passive movements throughout the investigation. Furthermore nail growth in the non-denervated digits of the involved hand was not affected. Finally, patients with similar lesions and treated in precisely the same way presented individual differences in which growth was slowed in some and not in others.

Rate of Growth in Median Nerve Lesions Associated with Ligation of the Brachial Artery.--In contrast to the occasional and slight slowing recorded after uncomplicated nerve injury, a pronounced retardation of nail growth was observed in the index finger and thumb in every patient who had sustained a combined neurovascular injury. With one exception the index finger was affected to a greater degree than the thumb.
In such complicated nerve injuries nail growth was slightly slowed in the non-denervated digits in the initial stages when the circulation of the peripheral parts of the limb was affected. As the circulation improved, however, this slowing was corrected and the rate then equalled that on the opposite side (Fig. 1).

It is noteworthy that the co-existence of a vascular and nerve injury was consistently associated with a retardation of nail growth which greatly exceeded that observed after the uncomplicated nerve injuries. It is difficult to determine to what extent this disturbance of nail growth is to be attributed to a vascular factor. That the marked retardation in the denervated digits is not due solely to circulatory embarrassment is suggested by the fact that the non-denervated digits rapidly recovered their original rate of growth when the circulation was restored whereas there was no such acceleration of growth in the denervated digits. Furthermore in Case L.H. it was observed that, 18 months after the injury when nerve function had been fully restored, the nail growth on the two sides was the same. At this time, however, there were signs of a persisting circulatory defect.

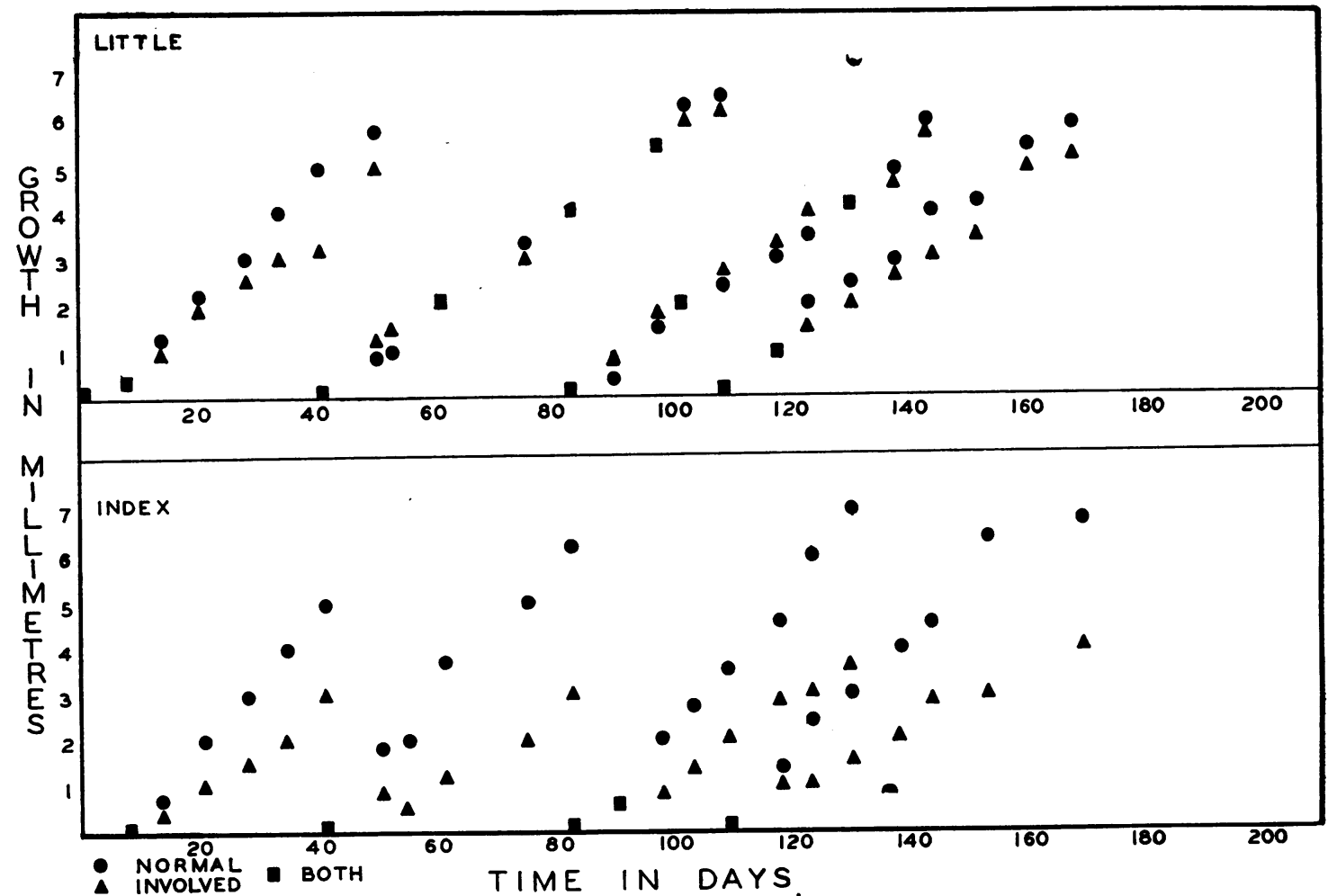

FIG. 1.-Graph to illustrate the rate of growth of the nails in a case of median nerve crush with ligation of the brachial artery. It will be noted that there is a marked difference in the rate of growth on the two sides for the index finger and virtually no difference for the little finger. 
There appeared to be a further slowing of nail growth during the winter months in two cases. Owing, however, to the limited number of patients available for observation, the data were insufficient for a precise assessment of the influence of the seasons on nail growth in denervated digits.

\section{Summary}

After uncomplicated nerve injuries nail growth in the denervated digits was retarded in some cases but not in others. When growth was affected the retardation was only slight.

When arterial ligation was superimposed on the nerve injury growth was consistently and markedly retarded in the denervated digits, greatly exceeding the slight slowing recorded after uncomplicated nerve injuries. There are three reasons why this greater slowing is not to be attributed solely to the vascular injury: (a) it outlasted the period of circulatory embarrassment ; $(b)$ the non-denervated digits of the involved hand were not affected; (c) complete nerve recovery resulted in an equality of growth in the involved and corresponding normal digits.

\section{REFERENCES}

Bernhardt, M. (1881). Virchows Arch., 86, 363.

Clark, W. E. Le Gros, and Buxton, L. H. D. (1938). Brit. J. Derm. Syph., 50, 221.

Head, H., and Sherren, J. (1905). Brain, 28, 116.

Mitchell, S. Weir (1872). "Injuries of Nerves and their Consequences." Philadelphia.

Sharpey-Schafer, E. (1931). Proc. roy. Soc. Eulinb., 51,8 . 